\title{
Measuring Lung Vessel Tree Growth During Development In Pediatric Patients
}

\author{
Aren Singh Saini, Shruti Siva Kumar, \& Walter O’Dell \\ University of Florida
}

Faculty mentor: Walter O’Dell, Department of Radiation Oncology

\begin{abstract}
Premature babies are often put on respirators due to the lack of development and functionality in their lungs. However, there is insufficient data regarding the point at which it is safe to take premature a baby off a respirator. This study investigates: how the pulmonary vasculature develops as the child grows, and can the exact time-point to take a patient off a respirator be determined. In this study, Chest CT scans were retrospectively gathered from pediatric patients at different follow-up times from the UF Shands Pulmonary Care Pediatric Center from 20052012. In-house software built upon the NIH ImageJ platform was used to count blood vessels as a function of size in each patient's lungs. Nine datasets were analyzed from subjects aged from 1 week to 22 years. It was observed that the number of vessels increased as a patient aged. However, the great spread of data prevented the study from being able to make additional inferences. Limitations of this study include the fact that patients were scanned only in instances of lung infections (which hinders the assessment of lung vasculature,) the number of repeated scans per patient was low, and the thickness of image slices and in-plane pixel resolution varied across scans, which may have affected the vessel count. Future extensions of this work include selecting a larger cohort of subjects with multiple follow-ups and similar imaging parameters, along with an age-matched control group.

Keywords: respirator, lung vasculature, lung development, premature birth, chest CT
\end{abstract}

\section{Introduction}

Every year, more than one in ten babies are born prematurely, leaving them highly vulnerable to infections and other ailment ("Preterm birth," 2018). One of the more serious complications is the lack of lung development and functionality. At the first breath, the entire vascular structure in the lungs start functioning as it received the entire output of circulation from the heart, allowing for gasses to be exchanged efficiently (Peng \& Morrisey, 2013). If a child is born prematurely, the lung vasculature is often not developed enough to receive proper circulation and efficient gas exchange. Often times, these children are put on respirators to increase the probability of survival. After some time, a patient is weaned off the respirator; most of the time after the first weaning trial, patients are able to breathe on their own ("Ventilator/Ventilator Support," n.d.). Although respirators increase the probability of survival, many complications can arise from it. Pneumonia is a very common side-effect of improper usage of respirators, and in order to properly use the respirator, physicians need to directly pinpoint when a patient's lungs are developed enough to work properly ("Ventilator/Ventilator Support," n.d.). 
There is little to no quantifiable data that specifically pinpoints when it is safe to take a child off a respirator along with an insufficient understanding of how breathing patterns are altered during development in preterm infants (premies). Some studies suggest that premies have a lower lung capacity and higher ventilation rate while others say that they can maintain functional lung volume at a high capacity (Latzin et al., 2009). Often times, premies' lung functionality is the same as that of young fullterm children and their breathing problems are not caught until they reach adolescence, such as asthma or some other sort of long-term lung complication (Blencowe et al., 2013). If the lung vasculature structure can be properly quantified, physicians may be able to accurately pinpoint whether a prematurely born patient's lungs are adequately developed enough to take him/her off a respirator. Thus, this study could provide important clinical guidance that can greatly improve the lives of children born prematurely.

\section{Hypothesis}

The overarching questions are: how do the pulmonary vasculature branch structure and branch radii increase during development and can it be determined whether a prematurely born patient's lungs are adequately developed enough to take him/her off a respirator. The immediate goal is to better document differences in lung development between pre-term and full-term patients from soon after birth into adolescence. By measuring the vascular structural trends over a period of time, it is expected that the development of patient's lungs can be determined quantitatively. With an increase in the age of the patient, there should be an increase in the number of branches and the radii size since the patients' total lungs sizes increase.
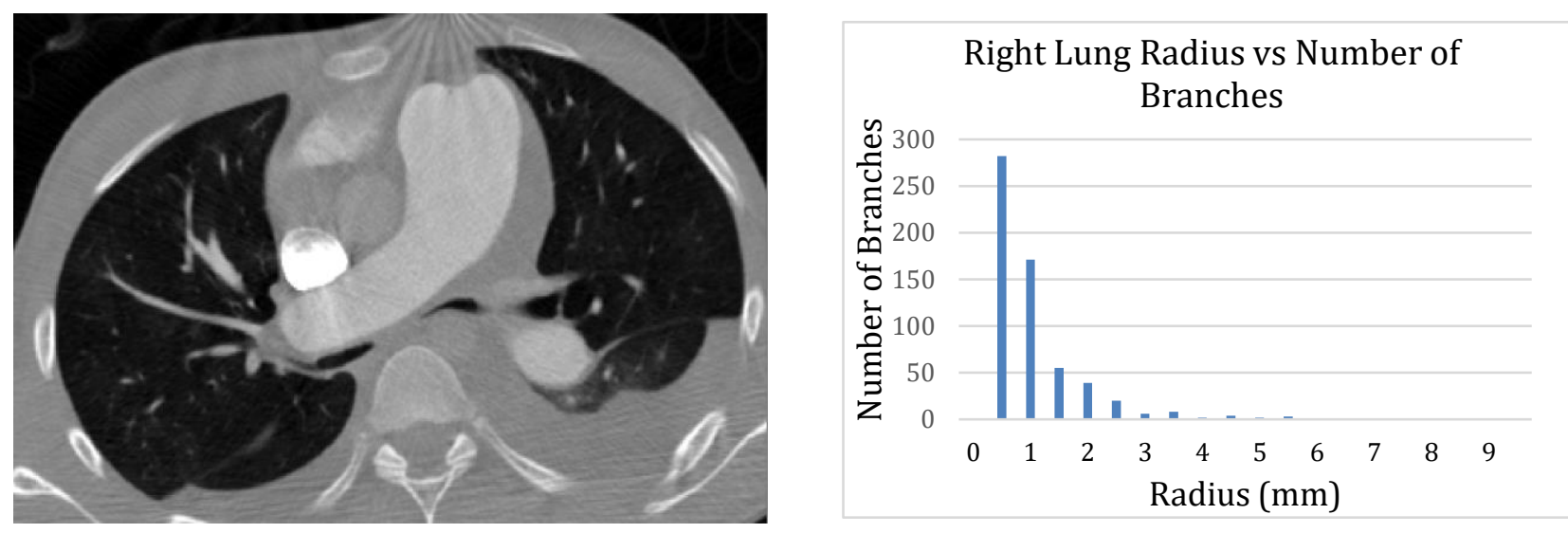

Figure 1: The image on the left is a representative high quality pediatric chest CT slice at the level of the superior heart. It has slice thickness of $1.25 \mathrm{~mm}$. As was often the case, this patient was affected by lung disease, most prominent here in the left hemi-lung, limiting our analysis to the right hemi-lung. The graph on the right is the branch radius histogram for this patient's right hemi-lung. The histogram exhibits the expected exponential increase in branch number for decreasing branch radius over the measurable size range. 


\section{Materials and Methods}

Lung CT scans at various time points during development were retrospectively gathered from patients seen at the UF Shands Pediatric Pulmonary Care Center from 2005-2012. In-house software built upon the NIH ImageJ platform was used to determine the number of blood vessel branches and their radii within the left or right hemi-lung in each patient. The program automatically identifies the hemi-lungs and applies semi-automatic segmentation using active contours/snakes to create hemi-lung masks. A region-growing method starting from a manually-selected seed point was used to extract the airway tree. The same approach was then applied to extract the pulmonary vessel trees. Skeletonization was applied to facilitate the traversing and characterizing of the tree structure. The radius and length of each branch were then computed as a function of age from birth. This approach was applied to nine pediatric chest CT datasets acquired from 1 week to 22 years of age. Patient age and pre-term status were also recorded when available.

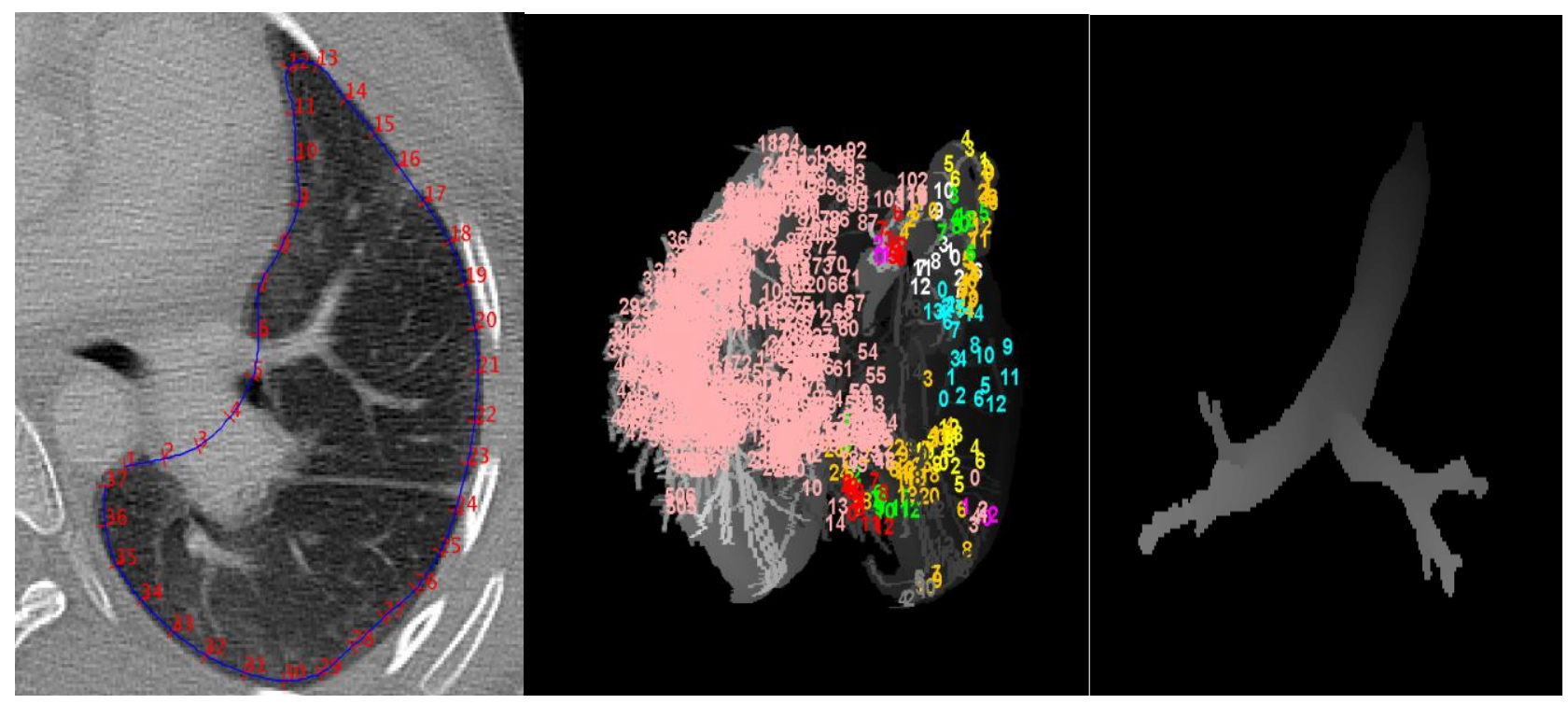

Figure 2. Lung contours, branch numbers, and airway vessel

The image on the left displays how the lungs were contoured. The contours were made so that all the large vessels in the lung were included within the lung mask. The middle image shows the numbering of the branches in the lung vessels. Finally, the last image is an airway vessel that was extracted so that the program can properly determine where the vessels are.

\section{Results}

By analyzing the data, we tried to determine whether if there is a common trend between age, lung radii, and the number of lung branches. As the radii of the lung vessels decreased, the number of branches increased exponentially. From the data, a typical patient had 100-200 branches within the 0-1 $\mathrm{mm}$ range. As a starting point, "the total number of branches with radii between 0 to $10 \mathrm{~mm}$ were computed. For some patients, such as the one showed in Figure 3B, the number of branches remained 
approximately constant over multiple scans. The number of subjects was too small to permit separating the data into pre-term and full-term cohorts, thus they are collectively shown in Figure 3B. The graph of Figure 1 shows a high-quality scan in a pediatric patient with a $1.25 \mathrm{~mm}$ slice thickness. However, that specific patient had no follow-up scans while the other patients had lower quality images, which made it difficult to compare the number of vessels per radii size range. When the total number of branches for each patient was graphed in Figure 3B, the data came out to be very scattered. The low resolution in scans and the variation in each patients' lungs must have contributed to this phenomenon. Figure 3A shows a patient that was scanned at ages 13 and 14 which had a higher number of branches overall, particularly in the $0-0.5 \mathrm{~mm}$ size range. By comparing multiple scans in one patient, we were able to see a clear increase in number of branches.
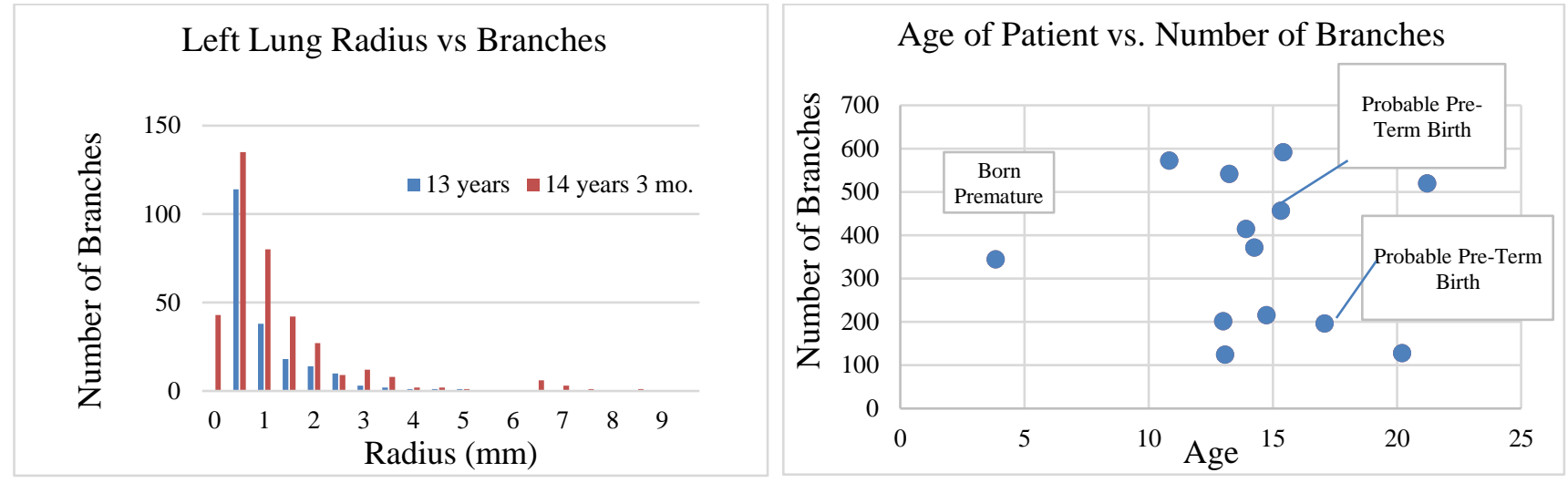

Figure 3. Number of branches for pediatric patients during development. [A-Left] shows a branch radius histogram for a representative patient at 13 and 14 years of age where slice thickness was $3 \mathrm{~mm}$ for both scans. [B-Right] shows total number of branches versus patient age (range $3-22$ years) compiled over 9 patients, with 4 providing 2 scans.

\section{Discussion}

Overall, an increase in the number of lung vessels within a few patients was seen, but it was not enough to make a substantial claim because the data points from all the patients' vessel branch numbers were scattered around graph when combined into one data pool. As seen through Figure 3A, there is an increase in the number of branches as the child ages. A slight increase was expected since lung sizes generally increase as a child grows (Rosenthal \& Bush, 2002). However, since the data points for the different patients were scattered in Figure 3B, determining the exact quantification of the trend between lung radii size and the number of branches over time was not feasible. From this data, it was not possible to determine if there was a correlation between the number of branches and the age of the patient.

The main problem with our analysis was that pediatric patients are typically scanned only when they have health issues with their lungs, such as infection or edema. According to a study on vascular 
changes in lungs, there are very few lung diseases that do not cause any change in the lung vascular structure (Kerr, 1970). When scanning very young patients, the scans often suffer as a result of a lower number of slices and increased motion artifact, thereby diminishing the accuracy of the estimates. When possible, data sets of unaffected lungs that had similar slice thicknesses, typically 1-4 mm, were comparatively analyzed. The images often have similar slice thicknesses (around 3mm) but lower resolutions. This problem arose throughout most of the scans with the patients.

Based on the findings of this initial study, a revised, updated IRB protocol overcoming many of the aforementioned limitations was drafted. It involves a large cohort (30) of scans from both the premies and control subjects from UF Shands Hospital. Only patients with >2 follow-up chest CT scans will be selected. The new protocol includes only patients that have been scanned since 2012, using more modern, high-quality CT imaging systems within the UF Department of Radiology. These scans will assist in quantifying the lung vascular structure in the near future as they can provide us with high quality scans that can be analyze thoroughly rather than the low-resolution ones from ten years ago. The scans received from the new protocol will be separated through the patients' gestational age, sex, and infection/disease. Grouping the data will allow us to see if there are any similarities or differences between certain types of patients. It will also easier to identify trends if we have multiple scans of the same patient to see how the number of branches increased/decreased over time. When comparing different patients, many confounding variables come into play as every patient has a different lung structure.

\section{Conclusion}

Our data showed a general trend for an increase in the number of branches over time, but with much variability. Much of this variability was due to differences in lung health issues of the patients and scan quality. Plans for future work are to increase the number of patients, increase the number of time-points per patient, and separate results based on patients' baseline issues (e.g, PAH, premature gestation, and infections). Ongoing work includes developing a way to match individual lung vessels and vessel trees over multiple scans to document the changes occurring on a vessel-by-vessel basis.

\section{Acknowledgements}

Funding for this work provided through the UF Research Scholars Program. 


\section{References}

Blencowe, H., Cousens, S., Chou, D., Oestergaard, M., Say, L., Moller, A., . . Lawn, J. (2013). Born Too Soon: The global epidemiology of 15 million preterm births. Reproductive Health, 10(Suppl 1).

doi:10.1186/1742-4755-10-s1-s2

Kerr, I. H. (1970). Vascular changes in the lungs on the plain radiograph of the chest. Postgraduate Medical Journal,46(531), 3-10. doi:10.1136/pgmj.46.531.3

Latzin, P., Roth, S., Thamrin, C., Hutten, G. J., Pramana, I., Kuehni, C. E., ... Frey, U. (2009). Lung volume, breathing pattern and ventilation inhomogeneity in preterm and term infants. PloS One, 4(2), e4635. https://doi.org/10.1371/journal.pone.0004635

O’Dell, W.G, Govindarajan, S.T., Salgia, A., Hegde, S., Prabhakaran, S., Finol, E.A., White, R.J., Traversing and labeling interconnected vascular tree structures from 3D medical images. In S. Ourselin \& M. A. Styner (Eds.), Proceedings of SPIE Medical Imaging 2014; (p. 90343C). doi:10.1117/12.2044140

Preterm birth. (2018, February 19). Retrieved September 18, 2018, from http://www.who.int/news-room/factsheets/detail/preterm-birth

Rosenthal, M., \& Bush, A. (2002). The growing lung: Normal development, and the long-term effects of pre- and postnatal insults. In Growing Up with Lung Disease: The Lung in Transition to Adult Life(pp. 1-24). UK: European Respiratory Monograph.

Ventilator/Ventilator Support | National Heart, Lung, and Blood Institute (NHLBI). (n.d.). Retrieved March 21, 2019, from https://www.nhlbi.nih.gov/health-topics/ventilatorventilator-support 